\title{
Emotion and motor preparation: A transcranial magnetic stimulation study of corticospinal motor tract excitability
}

\author{
STEPHen A. CoOmbes \\ University of Illinois, Chicago, Illinois \\ Christophe Tandonnet and Hakuei Fujiyama \\ University of Tasmania, Hobart, Tasmania, Australia \\ Christopher M. Janelle and James H. Cauraugh \\ University of Florida, Gainesville, Florida \\ AND \\ JEFFERY J. SUMMERS \\ University of Tasmania, Hobart, Tasmania, Australia
}

\begin{abstract}
In the present study, we examined whether preparing motor responses under different emotional conditions alters motor evoked potentials (MEPs) elicited by transcranial magnetic stimulation delivered to the motor cortex. Analyses revealed three findings: (1) Reaction times were expedited during exposure to unpleasant images, as compared with pleasant and neutral images; (2) force amplitude was greater during exposure to unpleasant images, as compared with pleasant and neutral images; and (3) MEPs were larger while participants viewed unpleasant images, as compared with neutral images. Hence, coupling the preparation of motor responses with the viewing of emotional images led to arousal-driven changes in corticospinal motor tract excitability, whereas movement speed and force production varied as a function of emotional valence. These findings demonstrate that the effects of emotion on the motor system manifest at varying sensitivity levels across behavioral and neurophysiological measures. Moreover, they validate the action readiness component of emotional experience by demonstrating that emotional states influence the execution of future movements but, alone, do not lead to overt movement.
\end{abstract}

The biphasic theory of emotion (Lang, Bradley, \& Cuthbert, 1997, 1998) posits that human emotion can be organized according to valence (i.e., appetitive or defensive) and intensity (i.e., arousal level). When engaged, appetitive or defensive systems affect the functioning brain, priming physiological adaptation and specific representations, associations, and action programs that correspond to the environmental context that has elicited the given emotion. The long-held view that emotions prime the human body for action (Ekman \& Davidson, 1994; Frijda, 1986, 2009; Frijda, Kuipers, \& ter Schure, 1989; Izard, 1994) has been supported by evidence showing that unpleasant cues activate the defensive system, which facilitates avoidance movements away from the cue (i.e., danger, fear) (although anger is one exception; Peterson, Shackman, \& Harmon-Jones, 2008), whereas pleasant cues activate the appetitive system and facilitate approach movements (i.e., excitement, food, sex; e.g., Chen \& Bargh, 1999; Rotteveel \& Phaf, 2004). Related work has shown that approach and avoidance movements are not directly linked to specific muscles (i.e., approach motivation $\neq$ flexors; avoidance motivation $\neq$ extensors) but are driven by the location of the active frame of reference (for detailed explanations, see Markman \& Brendl, 2005; Seibt, Neumann, Nussinson, \& Strack, 2008). Nevertheless, when the active frame of reference is one's self, considerable evidence demonstrates that the appetitive system primes approach/flexion movements and the defensive system primes avoidance/extension movements (Chen \& Bargh, 1999; Rotteveel \& Phaf, 2004). Accordingly, shorter reaction times (RTs) are evidenced when there is congruence between movement direction and motivational direction (e.g., defensive system + avoidance/ extension movements), as compared with when the pairing is incongruent (i.e., defensive system + approach/ flexion movements) (e.g., Chen \& Bargh, 1999). Building on this position, exposure to unpleasant images, as compared with pleasant and neutral images, has been shown to accelerate premotor RTs ${ }^{1}$ of upper limb ballistic extension movements (Coombes, Cauraugh, \& Janelle, 
2007a, 2007b) and increase the force amplitude of sustained extension contractions (Coombes, Cauraugh, \& Janelle, 2006). The assumption, therefore, is that emotional and motor processes are integrated and that this integration is reflected at the behavioral level and at the neurophysiological level. A developing body of work, described next, has examined how emotional states alter corticospinal motor tract (CST) excitability.

Transcranial magnetic stimulation (TMS) evidence has shown that varying levels of state- or trait-specific affect are associated with changes in CST excitability (Baumgartner, Willi, \& Jäncke, 2007; Hajcak et al., 2007; Oathes, Bruce, \& Nitschke, 2008; Oathes \& Ray, 2006; Oliveri et al., 2003; Schutter, Hofman, \& Van Honk, 2008; Tormos et al., 1997). Animal work has revealed strong connections between the limbic system and cingulate areas. Cingulate areas share reciprocal connections with the supplementary motor area (SMA), and the SMA projects to the primary motor cortex (M1) (Morecraft \& Van Hoesen, 1998). Building on this work, Oliveri et al. presented human participants with unpleasant and neutral images and used TMS to investigate whether the SMA serves as an interface between the emotion and motor systems. Oliveri and colleagues reported an increase in CST excitability during the presentation of unpleasant, as compared with neutral, images but identified two important caveats to their findings. First, the effect was present only when participants had to decide whether an image was unpleasant, as compared with a decision about whether "living" beings were present in an image (target stimuli differed between blocks). Second, facilitation was evidenced only when conditioning stimuli were applied to the SMA, as compared with the right premotor cortex and right M1, shortly before the test stimulation of the left M1. Recent TMS evidence has provided additional support for the position that unpleasant emotional states enhance action preparation (Oathes et al., 2008). What is not clear from these studies, however, is whether CST system excitability is driven by unpleasant valence or emotional arousal; in neither case was a pleasant condition included to control for a possible arousal effect. Hence, if emotional arousal was driving these alterations, pleasant and unpleasant emotional states should similarly affect/facilitate CST excitability. Conversely, if emotional valence was driving these alterations, pleasant and unpleasant emotional states should differentially influence CST excitability.

Additional evidence from Baumgartner and colleagues (Baumgartner et al., 2007) favors the postulate that arousal drives CST excitability under emotionally evocative conditions. Participants were presented with visual scenes and/or auditory stimuli to elicit fearful, sad, and happy emotional states. The stimuli were presented alone (i.e., image only, music only) or combined (image + music). When combined, the affective stimuli of the two stimulus modalities were always congruent (i.e., sad images + sad music). The authors hypothesized that combined conditions would elicit the greatest motor evoked potentials (MEPs) and that the fight/flight response would increase MEPs to unpleasant, as compared with pleasant, affective states. Increased MEPs were evidenced during the combined condition, as compared with the image-alone and the music-alone conditions. Importantly, no difference in CST excitability was evidenced between pleasant and unpleasant states in any single or combined condition. This similarity between valenced conditions supported the notion that CST excitability is modulated by emotional arousal, rather than by emotional valence.

Related work by Hajcak et al. (2007) measured CST excitability while participants passively viewed pleasant, unpleasant, and neutral images. The results were in agreement with Baumgartner et al. (2007) and supported an emotional arousal hypothesis. That is, greater MEPs were evidenced while participants viewed pleasant and unpleasant images, as compared with neutral images. The authors concluded that emotional-arousal-driven changes in CST excitability emerge even when no movement is prepared or executed by participants. It is noteworthy, however, that eliciting high levels of emotional arousal may mask potential valence effects. For instance, Schutter et al. (2008) reported that CST excitability increased when participants passively viewed fearful faces, as compared with happy and neutral faces. The important distinction between these and previous data is that emotional faces elicit an attenuated arousal response, as compared with emotional scenes (Britton, Taylor, Sudheimer, \& Liberzon, 2006). One interpretation of this discrepancy is that when elicited emotional arousal is high, valence effects are diminished, whereas when arousal effects are relatively low, valence effects emerge.

Taken together, although pleasant, unpleasant, and neutral conditions have not always been included in studies of CST excitability, there is a general consensus in the literature that viewing emotional scenes, as compared with neutral scenes, consistently leads to an increase in MEPs (Hajcak et al., 2007; Schutter et al., 2008). Although reliable, this pattern of activity has been demonstrated using experimental protocols in which the motor system was passive. Accordingly, whether active planning of overt motor behavior alters this relationship remains unknown. To address this gap in the literature, we sought to answer the following question: What are the effects on CST excitability when transient emotional states are coupled with the preparation of a voluntary motor action?

To assimilate previous TMS findings that have shown an arousal effect on CST excitability (Baumgartner et al., 2007; Hajcak et al., 2007) and behavioral findings that have shown a valence effect on motor output (Chen \& Bargh, 1999; Coombes et al., 2006, 2007a, 2007b; Coombes, Janelle, \& Duley, 2005; Rotteveel \& Phaf, 2004), in the present study, we examined how viewing emotional images while engaging the motor system via the preparation of a ballistic extension movement would modulate CST excitability. Pursuant to this aim and to replicate a previously used behavioral protocol (Coombes et al., 2007a, 2007b), we held the direction of the required movement constant (i.e., extension) and manipulated emotional state through the presentation of pleasant, unpleasant, and neutral images. The valence hypothesis suggests that when prepared extension movements (i.e., avoidance) are paired with unpleasant images that activate the defensive system, CST excitability will increase, RTs will decrease, and force 
production will increase, as compared with preparing the same movement during exposure to pleasant and neutral images. The alternative arousal hypothesis is that both pleasant and unpleasant images will lead to an increase in CST excitability, a decrease in RT, and an increase in force production, relative to neutral images. Previous behavioral evidence supports the valence hypothesis, whereas previous TMS evidence supports the arousal hypothesis. We predicted that the valence hypothesis would be supported across all behavioral and neurophysiological measures.

\section{METHOD}

\section{Participants}

Participants were 23 right-handed male volunteers (age: $M=$ 21.86 years, $S D=4.52$, range $=18-36$ ). All the participants were free of any neurological impairment, symptomatic cardiovascular disease, diabetes, or hypertension. Written informed consent was obtained prior to participation in the study, and ethics approval was obtained from the Human Research Ethics Committee Network. Participants with incomplete MEP, force, or self-reported valence and arousal data as a result of technical issues or failure to follow instructions were removed from all the analyses. Consequently, 8 participants were removed, resulting in a total of 15 participants (full data sets) being included in all the analyses (age: $M=21.60$, $S D=3.27$, range $=18-28$ ).

\section{Stimuli}

Each participant viewed 117 images taken from the International Affective Picture System (IAPS; Lang, Bradley, \& Cuthbert, 2005). ${ }^{2}$ Images were presented full screen on a 17 -in. monitor placed $70 \mathrm{~cm}$ in front of the participant, and each image was presented only once. The participants completed three experimental blocks, with each block composed of 39 images representing three categories (13 images per valence category: pleasant, unpleasant, and neutral). Images were chosen to maximally distinguish valence ratings between image categories and to maximize polarity between arousal ratings for emotional and neutral image categories. Normative ratings of valence and arousal were matched for trials on which TMS was delivered simultaneously with the auditory "go" cue (TMS condition) and trials on which the auditory cue was presented alone (no-TMS condition). ${ }^{3}$ Within experimental blocks, stimulus presentation order was randomized and counterbalanced, and block order was randomized between participants. Following completion of the experimental blocks, the participants self-reported valence and arousal ratings for the pictures, using a 9-point computerized version of the self-assessment manikin (Bradley \& Lang, 1994).

\section{Apparatus}

The participants were seated on a chair with their forearms supported and their right hand placed palm down under a pad attached to a force transducer (FTP-G-5000, Schaevitz). Hand position was adjusted to ensure that the center of the pad was between the hand joint and the proximal finger joint. The height of the force sensor was adjusted for each participant to ensure a permanent contact between the hand/fingers and the force sensor. The wrist and the hand were fixed with tape, and the hand and the fingers were further stabilized with foam pads positioned along the index finger and the little finger. Force signals were sampled at $2000 \mathrm{~Hz}$ and filtered offline with a $50-\mathrm{Hz}$ low-pass Butterworth filter.

The presentation of the TMS pulse and the auditory stimulus were simultaneous and triggered by the same computer. TMS was applied using a flat circular coil (9-cm diameter) connected to a Magstim 200 (Magstim, Dyfed, U.K.). The coil was held tangentially over the vertex to induce a posterior-anterior current flow in the left M1 (A-side up) and to elicit MEPs in the right and left extensor digitorum communis (EDC) and right flexor digitorum superficialis (FDS) muscles (Kobayashi \& Pascual-Leone, 2003). Electromyo- graphic (EMG) surface electrodes $(\mathrm{Ag} / \mathrm{AgCl}, 10 \mathrm{~mm}$ in diameter) were placed over the right and left EDC and right FDS in a bellytendon montage. Although activation in the right EDC was of particular interest, given its role as the prime mover in finger extension, a muscle contraction in one hand might modify the excitability of the homologous muscle in the other hand (e.g., Hess, Mills, \& Murray, 1986). The activity of all the recorded muscles was therefore monitored during the pre-TMS period to ensure that the MEP recorded in the target muscle was not contaminated by muscle activation of other muscles. The reference electrode was attached to the humeroradial joint of the elbow. EMG signals were amplified with a gain of 1,000, band-pass filtered $(10-500 \mathrm{~Hz})$, and sampled at $2000 \mathrm{~Hz}$ using a 16-bit AD system (Cambridge Electronic Design). The individual resting motor threshold (rMT) was determined as the lowest stimulus intensity that produced MEPs from the right EDC greater than $50 \mu \mathrm{V}$ on at least three out of five consecutive trials. The intensity of the TMS pulse was set at $120 \%$ of the rMT of the right EDC.

\section{Procedure}

During each of three experimental blocks, the participants viewed 39 images. Each image was visible for $5 \mathrm{sec}$, and intertrial intervals were $5 \mathrm{sec}$. During noncatch trials, an auditory stimulus $(80 \mathrm{~dB}$, 50 -msec duration) was presented $2-4 \mathrm{sec}$ after image onset. The participants were instructed to view each image for the entire time it was on the screen, to use image onset as a cue to get ready to move, and to respond as quickly as possible to each auditory stimulus by initiating a ballistic isometric extension of the wrist and fingers. Importantly, the instructions were applicable for all trials, since the goal was for the MEP to capture the integration of emotion and prepared voluntary motor processes. In each experimental block, TMS was delivered during the viewing of 12 pictures (4 TMS pulse trials per image category). TMS pulses were delivered simultaneously with the onset of the auditory stimulus (TMS condition). During each block, 24 images ( 8 per image category) were presented with auditory stimuli but without TMS (no-TMS condition). During each block, one image from each valence category was presented without an auditory stimulus or TMS. The total number of TMS trials per image category was 12 (4 trials in each block). The total number of no-TMS trials per image category was 24 ( 8 trials in each block). The total number of no-TMS plus no-auditory-cue trials per image category was 3 ( 1 trial in each block). Following the three experimental blocks, maximum voluntary contractions (MVCs) were calculated. The participants were asked to produce a brief wrist extension as hard as possible during a specified 10 -sec period while the force was displayed in real time on the screen in front of them. Five trials were completed, with $30 \mathrm{sec}$ of rest separating trials. The highest value from the 5 trials was taken as the MVC (MVC: $M=45.80 \mathrm{~N}, S E=$ 4.94). The participants then completed the picture-rating session.

\section{Data Reduction and Statistical Analyses}

RT and force data were calculated from all the trials on which auditory cues were presented (i.e., TMS and no-TMS trials). RTs reflected the time between acoustic cue onset and a $2-\mathrm{N}$ rise in force output (van der Lubbe \& Verleger, 2002). Force data for each trial were scored as the peak force amplitude reached during that trial, expressed as a percentage of the participants' MVC. MEP size was measured by calculating the maximum absolute value from $20 \mathrm{msec}$ post-TMS to $100 \mathrm{msec}$ post-TMS. Larger MEPs reflect greater CST excitability. MEPs were discarded if the root-mean square (RMS) of the EMG exceeded $15 \mu \mathrm{V}$ (Carson et al., 2004) during the $70 \mathrm{msec}$ immediately preceding the TMS pulse. No more than three trials were discarded per image condition per participant. A one-way repeated measures ANOVA revealed nonsignificant valence effects on the number of trials discarded $(p>.05)$. A total of $88 \%$ of the trials were included. Mean baseline EMG activity was the RMS of the EMG during a 70-msec window that ended $10 \mathrm{msec}$ prior to TMS delivery. Baseline activity from nondiscarded trials was averaged within each image condition and then analyzed to test for imagedriven changes in baseline EMG activity. 
To examine the potential influence of TMS delivery, RT, force, self-reported valence, and self-reported arousal were analyzed in separate 2 (condition: TMS, no TMS) $\times 3$ (image: pleasant, unpleasant, neutral) repeated measures ANOVAs. Baseline EMG data and MEP data were analyzed in separate one-way repeated measures ANOVAs (image). The critical $p$ value was set at .05 for all the analyses. If the sphericity assumption was violated, GreenhouseGeisser's degrees-of-freedom adjustment was applied to the critical $p$ values. Tukey-Kramer's follow-up procedure was used when appropriate.

\section{RESULTS}

\section{Self-Report Data}

Valence. Figure 1 shows mean self-reported valence for pleasant, unpleasant, and neutral images viewed during the TMS (black bars) and no-TMS (white bars) conditions. A significant main effect of image was evidenced $\left[F(2,28)=146.87, p<.05, \eta^{2}=.91\right]$. Follow-up analyses revealed that pleasant images were rated as more pleasant than were neutral and unpleasant images $(p s<.001)$ and neutral images were rated as more pleasant than were unpleasant images $(p<.001)$. The main effect of condition $\left[F(1,15)=4.32, p>.05, \eta^{2}=.27\right]$ and the interaction between condition and image $[F(2,248)=1.59, p>$ $\left..05, \eta^{2}=.12\right]$ were not significant. This demonstrates that the presence/absence of a TMS pulse during initial image presentation did not bias the subsequent valence rating of that image. Moreover, this finding validates the balanced manipulation of valenced images between the TMS and the no-TMS conditions.

Arousal. Figure 2 shows mean self-reported arousal for pleasant, unpleasant, and neutral images viewed during the TMS (black bars) and no-TMS (white bars) conditions. A significant main effect of image was evidenced $\left[F(2,28)=39.90, p<.05, \eta^{2}=.77\right]$, with follow-up tests revealing that pleasant and unpleasant images were rated as more arousing than were neutral images $(p \mathrm{~s}<.001)$ but were not different from each other $(p>.05)$. This

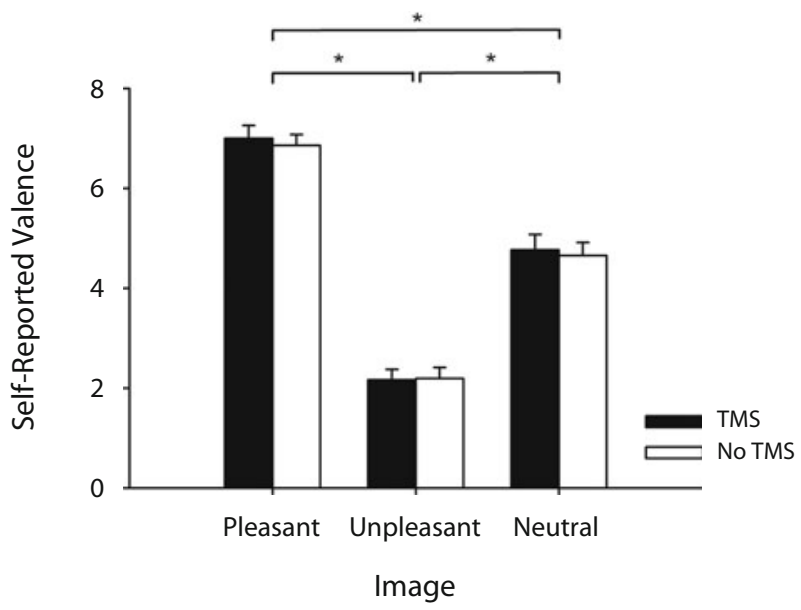

Figure 1. Mean self-reported valence ratings for pleasant, unpleasant, and neutral images viewed during trials on which transcranial magnetic stimulation (TMS) was delivered (black bars) and trials on which TMS was not delivered (white bars). The error bars represent $+1 S E$ from the mean. ${ }^{*} p<.05$.

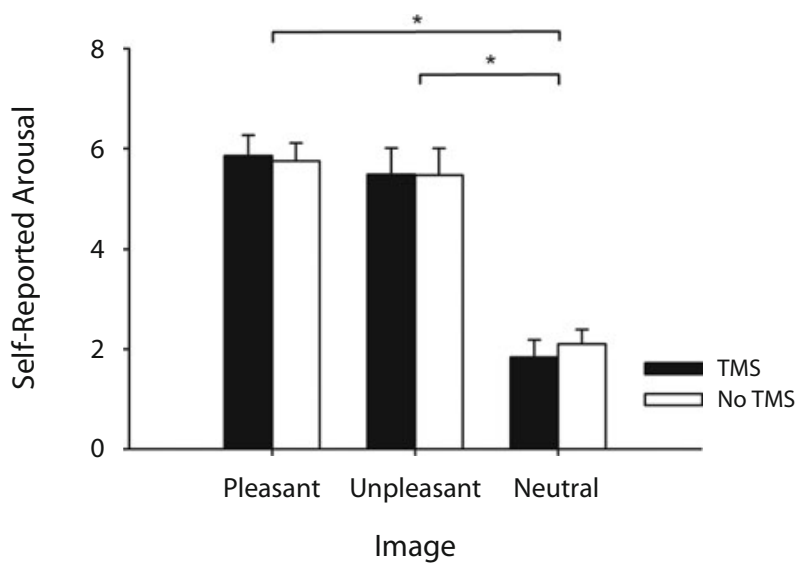

Figure 2. Across-participants mean self-reported arousal ratings for pleasant, unpleasant, and neutral images viewed during transcranial magnetic stimulation (TMS; black bars) and noTMS (white bars) conditions. The error bars represent $+1 S E$ from the mean. ${ }^{*} p<.05$.

pattern of findings was in agreement with the associated normative data that were used to guide image selection (Lang et al., 2005). Further confirmation of IAPS image selection was evidenced by a nonsignificant condition $X$ image interaction $\left[F(2,28)=2.26, p>.05, \eta^{2}=.16\right]$ and a nonsignificant main effect of condition $[F(1,14)=0.26$, $\left.p>.05, \eta^{2}=.02\right]$. Together, the latter findings demonstrate that images presented during TMS and no-TMS trials were matched for arousal within each image category and that the presence/absence of a TMS pulse during initial image presentation did not bias the subsequent arousal rating of that image.

\section{RT}

Figure 3 shows RT data during exposure to each image type during trials on which TMS pulses were delivered simultaneously with auditory cues (black bars) and during trials on which auditory cues were presented alone (i.e., no TMS; white bars). A two-way repeated measures ANOVA on RT indicated a significant main effect of image $\left[F(2,28)=7.45, p<.05, \eta^{2}=.35\right]$. Post hoc analyses for the image main effect revealed that RTs were shorter during exposure to unpleasant, as compared with neutral, images $(p=.002)$, and this finding was consistent for $87 \%$ (13/15) of the participants. Shorter RTs were found during exposure to unpleasant, as compared with pleasant, images $(p=.029)$, and this pattern was present for $80 \%(12 / 15)$ of the participants. The follow-up test between pleasant and neutral images was not significant $(p=.269)$.

A significant main effect of condition was also evidenced $\left[F(1,14)=17.84, p<.05, \eta^{2}=.56\right]$, which was driven by shorter RTs during TMS, as compared with no-TMS, trials (TMS, $M=237.30, S E=17.84$; no TMS, $M=280.02, S E=16.89)$. The condition $\times$ image interaction was not significant $[F(2,28)=2.01, p>.05$, $\eta^{2}=.13$ ], confirming that the delivery of TMS pulses did not interact with image type to influence RT. Indeed, as is shown in Figure 3, the RT patterns across image type were similar for TMS and no-TMS trials. 


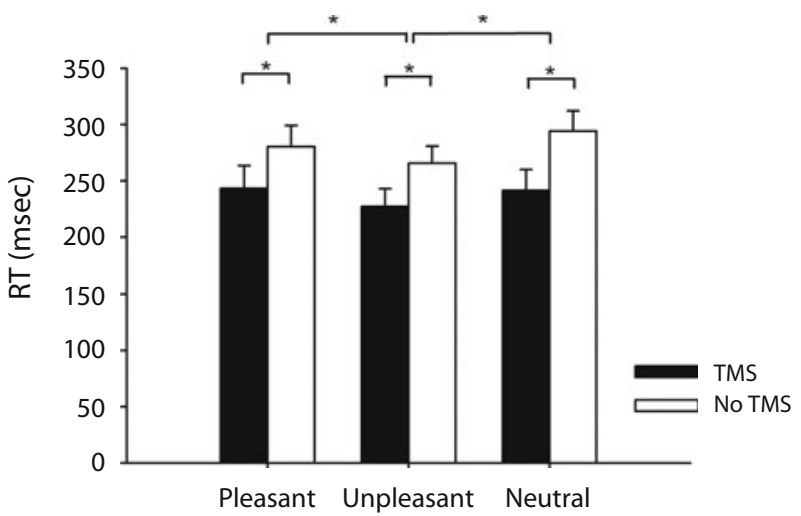

Image

Figure 3. Across-participants mean reaction time (RT) data during exposure to each image type during trials on which TMS pulses were delivered simultaneously with auditory cues (black bars) and during trials on which auditory cues were presented alone (white bars). The error bars represent $+1 S E$ from the mean. " $p<.05$.

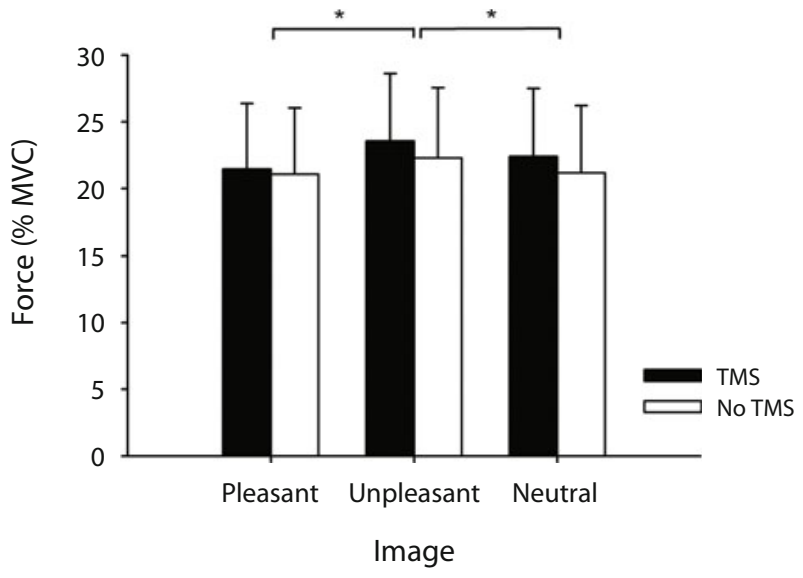

Figure 4. Across-participants mean force production, represented as a percentage of participants' maximum voluntary contractions (MVCs) during exposure to pleasant, unpleasant, and neutral images for trials on which transcranial magnetic stimulation (TMS) was (black bars) and was not (white bars) delivered with the auditory cue. The error bars represent $+1 S E$ from the mean. " $p<.05$.

\section{Force Production}

Figure 4 shows how force output (represented as a percentage of the participants' MVC) varied during exposure to pleasant, unpleasant, and neutral images for trials on which TMS was (black bars) and was not (white bars) delivered with the auditory cue. A main effect of image was evidenced on force output $\left[F(2,28)=8.05, p<.05, \eta^{2}=\right.$ $.37]$, with Tukey-Kramer's test showing greater force production during unpleasant than during pleasant images $(p=.005)$. This pattern of activity was evidenced in $60 \%$ $(9 / 15)$ of the participants. Furthermore, higher force production was evidenced during the presentation of unpleasant, as compared with neutral, images $(p=.012)$, and $73 \%$ of the participants displayed this pattern. Force pro- duction during exposure to pleasant and neutral images was not significantly different $(p=.182)$. A main effect of condition $\left[F(1,14)=0.26, p>.05, \eta^{2}=.02\right]$ and the condition $\times$ image interaction $[F(2,28)=2.26, p>.05$, $\left.\eta^{2}=.16\right]$ were not significant.

\section{Baseline EMG Data}

Analyses comparing the RMS of baseline EMG activity revealed nonsignificant effects of image for each muscle of interest [right EDC, $F(2,28)=1.90, p>.05, \eta^{2}=.12$; left EDC, $F(2,28)=2.00, p>.05, \eta^{2}=.13$; right FDS, $\left.F(2,28)=2.35, p>.05, \eta^{2}=.14\right]$. This finding demonstrates that changes in affective state and motor preparation do not alter levels of EMG activity recorded with surface electrodes and further confirms that any potential differences in MEP amplitude between image and condition were not confounded by baseline EMG activity.

\section{MEPs}

Right EDC. Figure 5 shows mean MEP amplitude during exposure to pleasant, unpleasant, and neutral images. Analysis of MEP amplitude revealed a significant effect of image $\left[F(2,28)=4.49, p<.05, \eta^{2}=.24\right]$. Followup analyses indicated that MEPs were greater while participants viewed unpleasant, as compared with neutral, images $(p=.01)$. This pattern of activity was evidenced in $87 \%(13 / 15)$ of the participants. A marginal difference was evidenced between pleasant and neutral images $(p=.078)$, with the trend suggesting greater MEPs during exposure to pleasant images. Seventy-three percent of the participants showed this pattern of activity. MEPs during unpleasant and pleasant images were not statistically different ( $p=.279$ ). Finally, we ran correlation analyses to examine the relation between RT, force, and MEPs for pleasant, unpleasant, and neutral images for the TMS and no-TMS conditions (e.g., TMS: pleasant RT vs. pleasant MEP). No significant effects were revealed (all $p \mathrm{~s}>.05)$.

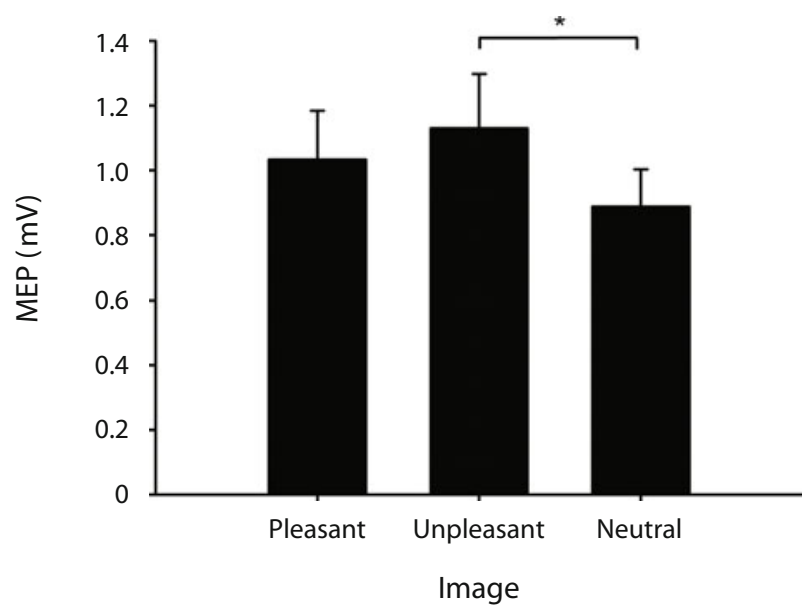

Figure 5. Mean motor evoked potential (MEP) data from the right extensor digitorum communis muscle averaged across participants during exposure to pleasant, unpleasant, and neutral images. The error bars represent $+1 S E$ from the mean. ${ }^{*} p<.05$. 
Left EDC/right FDS. No significant effect of image was evidenced on MEPs recorded from the left EDC $\left[F(1.43,20.11)=2.23, p>.05, \eta^{2}=.14\right]$ or the right $\operatorname{FDS}\left[F(1.37,19.307)=2.42, p>.05, \eta^{2}=.15\right]$.

\section{DISCUSSION}

Previous evidence has shown increased CST excitability during the passive viewing of pleasant and unpleasant images, as compared with neutral images (Hajcak et al., 2007). However, additional evidence shows that premotor RTs recorded during extension movements are shorter during exposure to unpleasant images, as compared with pleasant and neutral images (Coombes et al., 2007a, 2007b). We examined whether this valence-driven change in motor output would be reflected at the neurophysiological level by a concomitant change in CST excitability. The present study offers three contributions. First, although baseline EMG activity did not differ between emotional image conditions, MEP findings adhered to a pattern that reflected the emotional arousal (rather than valence) under which they were elicited. Second, RT and force measures varied as a function of emotional valence. Third, TMS delivery did not interact with image content to alter RT, force, or self-reported ratings but did lead to expedited RTs, irrespective of image content. Together, these findings demonstrate that the effects of emotion on the motor system manifest at varying sensitivity levels across behavioral and neurophysiological measures. Moreover, they validate the action readiness component of emotional experience by demonstrating that emotional states influence the execution of future movements but, alone, do not lead to overt movement. Each of these findings will be discussed in greater detail next.

\section{Arousal Effects on CST Excitability}

The present CST excitability findings corroborate those of Hajcak et al. (2007) in that MEP amplitude during exposure to pleasant and unpleasant images was similar, but each was strongly (unpleasant $>$ neutral) and marginally (pleasant $>$ neutral) different from that for neutral images. Our findings, therefore, were generally compliant with previous TMS evidence that has demonstrated that, when emotion images are viewed passively, emotional arousal, rather than emotional valence, potentiates CST excitability (Baumgartner et al., 2007; Hajcak et al., 2007). The present findings add further support to the position that activation of the fight/flight response does not increase excitability of the CST above and beyond activity associated with pleasant stimuli. Furthermore, they demonstrate that although the viewing of emotion images alone does not alter baseline EMG activity (measured with surface electrodes), it does alter CST excitability during the initiation of overt movement. The action readiness component of emotional experience (e.g., Frijda, 2009), therefore, does not appear to manifest in surface EMG. Instead, the present evidence suggests that brain regions central to the human motor system must overlap with and/or share structural or functional linkages with areas that are central to the expression of transient emotional states (Carretié,
Albert, López-Martín, \& Tapia, 2009). Importantly, action readiness resulting from the integration of emotion and motor processes does not appear to manifest peripherally until overt movements are initiated.

Emotional arousal/intensity, as induced by gustatory, olfactory, or visual emotional stimuli, has been shown to be positively correlated with neuronal activity in the amygdala (Anderson et al., 2003; Sabatinelli, Bradley, Fitzsimmons, \& Lang, 2005; Small et al., 2003) and the visual cortex (Sabatinelli et al., 2005). Related work has also shown that arousal is correlated with neuronal activity in the ventrolateral prefrontal cortex and dorsomedial prefrontal cortex, whereas valence correlates with neuronal activity in the ventromedial prefrontal cortex and dorsolateral prefrontal cortex (Anders, Lotze, Erb, Grodd, \& Birbaumer, 2004; Dolcos, LaBar, \& Cabeza, 2004; Grimm et al., 2006). The present findings provide further evidence that an emotionally driven arousal response impacts the motor system, and this integration is reflected in emotion-driven changes in CST excitability. However, despite a growing database that addresses the influence of emotion on motor function at the behavioral and neurophysiological level (e.g., Coombes et al., 2006, 2007a, 2007b; Coombes, Gamble, Cauraugh, \& Janelle, 2008; Coombes et al., 2005; Oathes et al., 2008; Oathes \& Ray, 2006) and a well-established body of literature that has focused on the neural correlates of emotional valence and emotional arousal (e.g., Grimm et al., 2006; Sabatinelli et al., 2005), our understanding of the mechanism(s) that integrate emotional and motor processes in the human brain remains vague.

Emerging comparative work with primates and rodents, however, suggests that the emotion and motor systems are integrated at the level of the basal ganglia (BG; Groenewegen, Wright, Beijer, \& Voorn, 1999; Haber, Fudge, \& McFarland, 2000; Haber, Kim, Mailly, \& Calzavara, 2006; McFarland \& Haber, 2002) via two distinct mechanisms. A first possibility is that the dendrites and axons within each structure of the BG often cross functional domains, allowing dendrites from one region to invade an adjacent functional region. Haber (2003) has advocated a second option, however, suggesting that information from distinct cortical regions could be processed separately and in parallel through functionally related neurons. Accordingly, information between the parallel cortico-striatal pathways could be integrated in two potential ways: (1) by spiraling connections between the midbrain dopamine cells and the striatum (striato-nigro-striatal network) and (2) via thalamo-cortico-thalamic projections. A third mechanism, also developed from animal work, suggests that the cingulate motor cortex forms a strategic cortical entry point for limbic influence on the voluntary motor system (Morecraft \& Van Hoesen, 1998). Determining whether these mechanisms map to the human brain, whether they function independently, collectively, or exclusively, and whether they underlie the arousal-driven changes in CST excitability remains a daunting but intriguing task for future research.

Strict adherence to an arousal interpretation of our data would have been permitted had MEPs also been greater 
during pleasant, as compared with neutral, images. Although a marginal trend supported this predicted pattern of activity (which was present in 11/15 of the participants), we suggest that the prepared extension movement (i.e., away from the body) was incongruent with the approach motivational system that was activated by the pleasant image, and this incongruence may account for the finding that pleasant cues did not elicit a response significantly different from that elicited by neutral cues. Nevertheless, the lack of a difference between MEPs during exposure to unpleasant and pleasant images and the marginal trend that distinguished pleasant from neutral images suggest that arousal, rather than valence, was the primary modulator of CST excitability. We also acknowledge that sample size may have been a contributing factor. However, strong effects of emotion on RT and force measures corroborated previous evidence, and Hajcak et al. (2007) have previously shown arousal effects on CST excitability in a similar sample size $(N=16)$. Furthermore, although selfreported arousal ratings for pleasant images in the present study were less than the corresponding normative ratings and less than those reported by Hajcak et al. (present study, TMS, $M=5.58$, and no TMS, $M=5.47$; normative ratings, $M=6.48$; Hajcak, $M=6.33$ ), they were greater than arousal ratings for unpleasant images (present study, TMS, $M=5.23$; no TMS, $M=5.16$ ). Moreover, Hajcak et al. reported high levels of arousal for unpleasant images $(M=8.40)$, as compared with pleasant images $(M=6.33)$, but found no differences between MEPs during pleasant and unpleasant conditions. Hence, although our arousal ratings were marginally suppressed, they were similar for pleasant and unpleasant images, which suggests that the marginally significant finding for pleasant versus neutral MEPs was not related to the manipulation of arousal in the pleasant condition.

\section{Valence Effects on RT and Force}

In contrast to the arousal pattern evidenced in the MEP data, RT and force data followed a valence-specific pattern of activity and replicated previous evidence by revealing a facilitation effect when unpleasant images, as compared with pleasant and neutral images, were combined with extension movements (Chen \& Bargh, 1999; Coombes et al., 2007a, 2007b; Rotteveel \& Phaf, 2004). This finding supports the long-held notion that emotions prime the human body for action (Ekman \& Davidson, 1994; Frijda, 1986, 2009; Frijda et al., 1989; Izard, 1994). Although generalization from the present data is restricted to extension movements, the results were clear in showing facilitation of movement speed and force production during exposure to unpleasant stimuli, as compared with pleasant and neutral ones. Given the equivocal effects of gender on emotional reactivity and motor output (Christou, Jakobi, Critchlow, Fleshner, \& Enoka, 2004; Coombes et al., 2008), we chose to focus the present study on males. It is important, therefore, that future work should extend the present findings by examining the potential interaction between emotion, gender, and CST excitability.

No significant relation was evidenced between MEPs and RT and force. Previous evidence has demonstrated that MEP amplitude does not always correlate highly with force amplitude when force production is relatively low (Perez \& Cohen, 2009). Given the relatively low forces executed in the present study ( $\approx 20 \%$ of MVC) and the relatively small differences between force levels $(\approx 2 \%$ of MVC) across pleasant, unpleasant, and neutral images, it is possible that the MEP measure was not sensitive enough to capture this distinction. In addition, the relation between MEP and RT appears to be more related to the interval between TMS pulse delivery and the initiation of movement. The TMS pulse was delivered simultaneously with the auditory cue. Accordingly, the shorter RTs during TMS, as compared with no-TMS, trials were consistent with the results of previous studies, which have demonstrated that shorter interstimulus intervals lead to shorter RTs (Masur, Schneider, Papke, \& Oberwittler, 1996; Nikolova, Pondev, Christova, Wolf, \& Kossev, 2006; Pascual-Leone et al., 1992). In the present study, the facilitation effect of the single TMS pulse was approximately $40 \mathrm{msec}$, and this effect was stable across pleasant, unpleasant, and neutral images. We interpret these findings as reflective of the intersensory facilitation effect, in which the presence of multiple warning signals from different sensory modalities (i.e., acoustic cue and TMS pulse) results in a larger facilitation of RT than that induced by a single warning signal (i.e., acoustic cue alone) (Diederich \& Colonius, 2008; Nickerson, 1973; Romaiguère, Possamaï, \& Hasbroucq, 1997).

Behavioral and neurophysiological findings were underpinned by robust self-report data, permitting confidence in a converging operations approach that suggests that whereas emotional arousal modulates CST excitability, emotional valence modulates RT and force production. Although we do acknowledge that this finding may be reflective of the sensitivity of the different measures used, the present findings represent the first evidence of a dissociation between the impact of emotion on the motor system at the neurophysiological and behavioral levels. Importantly, these findings caution against generalizing the effects of emotion across varying levels of motor system function.

We hope that the present evidence will motivate future researchers to systematically pursue important questions through several different protocols and measures. First, replicating the present study but using flexion rather than extension movements should, in principle, lead to a bias toward greater MEPs during exposure to pleasant images. Likewise, a pure emotional arousal effect should be evidenced if the manipulated motor task is nondirectional (i.e., a pinch grip). The latter prediction is based on previous work that has shown that when a precision grip force is to be maintained to a target level, the viewing of neutral images leads to a significant decay in force production, whereas pleasant and unpleasant images lead to a relative maintenance of force production (Coombes et al., 2008). Second, these findings have implications for clinical populations. For instance, future researchers are encouraged to build on previous work that has linked motor abnormalities with affective disorders (Oathes \& Ray, 2006; Wassermann, Greenberg, Nguyen, \& Murphy, 2001) by investigating the $(\mathrm{ab})$ normal functioning of the integra- 
tive mechanism(s) that may be at the core of symptoms related to depression and impulsivity. Third, building on primate work (Haber, 2003; Morecraft \& Van Hoesen, 1998), a key goal for future basic scientific research is to identify the mechanism(s) in humans that integrate emotional and motor processes. Armed with such knowledge, novel pharmacological and behavioral treatments can be developed to aid in the amelioration of both motor and emotional disorders.

\section{AUTHOR NOTE}

This research was supported in part by National Institute of Health Grants F32-MH-083424, R03-MH-70678, and R03-HD-044534 and American Heart Association Grant 00061194 . We thank Michael I. Garry for his assistance in designing the study and for his insightful comments during manuscript preparation. Correspondence concerning this article should be addressed to S. A. Coombes, Department of Kinesiology and Nutrition, University of Illinois, 1919 West Taylor, 650 AHSB (M/C 994), Chicago, IL 60612 (e-mail: scoombes@uic.edu).

\section{REFERENCES}

Anders, S., Lotze, M., Erb, M., Grodd, W., \& Birbaumer, N. (2004). Brain activity underlying emotional valence and arousal: A responserelated fMRI study. Human Brain Mapping, 23, 200-209.

Anderson, A. K., Christoff, K., Stappen, I., Panitz, D., GhahreMANI, D. G., Glover, G., ET AL. (2003). Dissociated neural representations of intensity and valence in human olfaction. Nature Neuroscience, 6, 196-202.

Basmajian, J. V., \& De Luca, C. J. (1985). Muscles alive: Their functions revealed by electromyography (5th ed.). Baltimore: Williams \& Wilkins.

Baumgartner, T., Willi, M., \& JÄNCKe, L. (2007). Modulation of corticospinal activity by strong emotions evoked by pictures and classical music: A transcranial magnetic stimulation study. NeuroReport, 18, 261-265.

Bradley, M. M., \& Lang, P. J. (1994). Measuring emotion: The selfassessment manikin and the semantic differential. Journal of Behavior Therapy \& Experimental Psychiatry, 25, 49-59.

Britton, J. C., Taylor, S. F., Sudheimer, K. D., \& Liberzon, I. (2006). Facial expressions and complex IAPS pictures: Common and differential networks. NeuroImage, 31, 906-919.

Carretié, L., Albert, J., López-Martín, S., \& TApia, M. (2009). Negative brain: An integrative review on the neural processes activated by unpleasant stimuli. International Journal of Psychophysiology, 71, 57-63.

Carson, R. G., Riek, S., Mackey, D. C., Meichenbaum, D. P., Willms, K., Forner, M., \& Byblow, W. D. (2004). Excitability changes in human forearm corticospinal projections and spinal reflex pathways during rhythmic voluntary movement of the opposite limb. Journal of Physiology, 560, 929-940.

Chen, M., \& BARgh, J. A. (1999). Consequences of automatic evaluation: Immediate behavioral predispositions to approach or avoid the stimulus. Personality \& Social Psychology Bulletin, 25, 215-224.

Christou, E. A., Jakobi, J. M., Critchlow, A., Fleshner, M., \& ENOKA, R. M. (2004). The 1- to 2-Hz oscillations in muscle force are exacerbated by stress, especially in older adults. Journal of Applied Physiology, 97, 225-235.

Coombes, S. A., Cauraugh, J. H., \& Janelle, C. M. (2006). Emotion and movement: Activation of defensive circuitry alters the magnitude of a sustained muscle contraction. Neuroscience Letters, 396, 192-196.

Coombes, S. A., Cauraugh, J. H., \& Janelle, C. M. (2007a). Dissociating motivational direction and affective valence: Specific emotions alter central motor processes. Psychological Science, 18, 938-942.

Coombes, S. A., Cauraugh, J. H., \& Janelle, C. M. (2007b). Emotional state and initiating cue alter central and peripheral motor processes. Emotion, 7, 275-284.

Coombes, S. A., Gamble, K. M., Cauraugh, J. H., \& Janelle, C. M. (2008). Emotional states alter force control during a feedback occluded motor task. Emotion, 8, 104-113.
Coombes, S. A., Janelle, C. M., \& Duley, A. R. (2005). Emotion and motor control: Movement attributes following affective picture processing. Journal of Motor Behavior, 37, 425-436.

Diederich, A., \& Colonius, H. (2008). Crossmodal interaction in saccadic reaction time: Separating multisensory from warning effects in the time window of integration model. Experimental Brain Research, 186, 1-22.

Dolcos, F., LaBar, K. S., \& Cabeza, R. (2004). Dissociable effects of arousal and valence on prefrontal activity indexing emotional evaluation and subsequent memory: An event-related fMRI study. NeuroImage, 23, 64-74.

Ekman, P., \& Davidson, R. J. (EDs.) (1994). The nature of emotion: Fundamental questions. New York: Oxford University Press.

FriJdA, N. H. (1986). The emotions. Cambridge: Cambridge University Press.

FrIJDA, N. H. (2009). Emotion experience and its varieties. Emotion Review, 1, 264-271.

FriJda, N. H., KuiPers, P., \& Ter Schure, E. (1989). Relations among emotion, appraisal, and emotional action readiness. Journal of Personality \& Social Psychology, 57, 212-228.

Grimm, S., Schmidt, C. F., Bermpohl, F., Heinzel, A., Dahlem, Y., WYss, M., ET AL. (2006). Segregated neural representation of distinct emotion dimensions in the prefrontal cortex: An fMRI study. NeuroImage, 30, 325-340.

Groenewegen, H. J., Wright, C. I., Beijer, A. V., \& Voorn, P. (1999). Convergence and segregation of ventral striatal inputs and outputs. In J. F. McGinty (Ed.), Advancing from the ventral striatum to the extended amygdala: Implications for neuropsychiatry and drug abuse (Annals of the New York Academy of Sciences, Vol. 877, pp. 49-63). New York: New York Academy of Sciences.

HABER, S. N. (2003). The primate basal ganglia: Parallel and integrative networks. Journal of Chemical Neuroanatomy, 26, 317-330.

Haber, S. N., Fudge, J. L., \& McFarland, N. R. (2000). Striatonigrostriatal pathways in primates form an ascending spiral from the shell to the dorsolateral striatum. Journal of Neuroscience, 20, 2369-2382.

Haber, S. N., Kim, K.-S., Mailly, P., \& Calzavara, R. (2006). Rewardrelated cortical inputs define a large striatal region in primates that interface with associative cortical connections, providing a substrate for incentive-based learning. Journal of Neuroscience, 26, 8368-8376.

Hajcak, G., Molnar, C., George, M. S., Bolger, K., Koola, J., \& NAHAS, Z. (2007). Emotion facilitates action: A transcranial magnetic stimulation study of motor cortex excitability during picture viewing. Psychophysiology, 44, 91-97.

Hess, C. W., Mills, K. R., \& Murray, N. M. F. (1986). Magnetic stimulation of the human brain: Facilitation of motor responses by voluntary contraction of ipsilateral and contralateral muscles with additional observations on an amputee. Neuroscience Letters, 71, 235-240.

IZARD, C. E. (1994). Innate and universal facial expressions: Evidence from developmental and cross-cultural research. Psychological Bulletin, 115, 288-299.

Kobayashi, M., \& Pascual-Leone, A. (2003). Transcranial magnetic stimulation in neurology. Lancet Neurology, 2, 145-156.

Lang, P. J., Bradley, M. M., \& Cuthbert, B. N. (1997). Motivated attention: Affect, activation, and action. In P. J. Lang, R. F. Simons, \& M. T. Balaban (Eds.), Attention and orienting: Sensory and motivational processes (pp. 97-135). Mahwah, NJ: Erlbaum.

Lang, P. J., Bradley, M. M., \& Cuthbert, B. N. (1998). Emotion, motivation, and anxiety: Brain mechanisms and psychophysiology. Biological Psychiatry, 44, 1248-1263.

Lang, P. J., Bradley, M. M., \& Cuthbert, B. N. (2005). International affective picture system (IAPS): Affective ratings of pictures and instruction manual (Tech. Rep. No. A-8). Gainesville: University of Florida.

Li, S., Stevens, J. A., Kamper, D. G., \& Rymer, W. Z. (2005). The movement-specific effect of motor imagery on the premotor time. Motor Control, 9, 119-128.

Markman, A. B., \& Brendl, C. M. (2005). Constraining theories of embodied cognition. Psychological Science, 16, 6-10.

Masur, H., Schneider, U., Papke, K., \& Oberwittler, C. (1996). Variation of reaction time can be reduced by the time locked application of magnetic stimulation of the motor cortex. Electromyography \& Clinical Neurophysiology, 36, 495-501.

McFarland, N. R., \& Haber, S. N. (2002). Thalamic relay nuclei of the 
basal ganglia form both reciprocal and nonreciprocal cortical connections, linking multiple frontal cortical areas. Journal of Neuroscience, 22, 8117-8132.

Morecraft, R. J., \& Van Hoesen, G. W. (1998). Convergence of limbic input to the cingulate motor cortex in the rhesus monkey. Brain Research Bulletin, 45, 209-232.

NICKERSON, R. S. (1973). Intersensory facilitation of reaction time: Energy summation or preparation enhancement? Psychological Review, 80, 489-509.

Nikolova, M., Pondev, N., Christova, L., Wolf, W., \& Kossev, A. R. (2006). Motor cortex excitability changes preceding voluntary muscle activity in simple reaction time task. European Journal of Applied Physiology, 98, 212-219.

OAthes, D. J., BRUCE, J. M., \& NitschKe, J. B. (2008). Worry facilitates corticospinal motor response to transcranial magnetic stimulation. Depression \& Anxiety, 25, 969-976.

OATHES, D. J., \& RAY, W. J. (2006). Depressed mood, index finger force and motor cortex stimulation: A transcranial magnetic stimulation (TMS) study. Biological Psychology, 72, 271-277.

Oliveri, M., Babiloni, C., Filippi, M. M., Caltagirone, C., BabiLONI, F., CiCINELli, P., ET AL. (2003). Influence of the supplementary motor area on primary motor cortex excitability during movements triggered by neutral or emotionally unpleasant visual cues. Experimental Brain Research, 149, 214-221.

Pascual-Leone, A., Valls-Sole, J., Wassermann, E. M., BrasilNeto, J., Cohen, L. G., \& Hallett, M. (1992). Effects of focal transcranial magnetic stimulation on simple reaction time to acoustic, visual and somatosensory stimuli. Brain, 115, 1045-1059.

Perez, M. A., \& Cohen, L. G. (2009). Scaling of motor cortical excitability during unimanual force generation. Cortex, 45, 1065-1071.

Peterson, C. K., Shackman, A. J., \& Harmon-Jones, E. (2008). The role of asymmetrical frontal cortical activity in aggression. Psychophysiology, 45, 86-92.

Romaiguère, P., Possamaï, C.-A., \& HasbroucQ, T. (1997). Motor cortex involvement during choice reaction time: A transcranial magnetic stimulation study in man. Brain Research, 755, 181-192.

Rotteveel, M., \& Phaf, R. H. (2004). Automatic affective evaluation does not automatically predispose for arm flexion and extension. Emotion, 4, 156-172.

Sabatinelli, D., Bradley, M. M., Fitzsimmons, J. R., \& Lang, P. J. (2005). Parallel amygdala and inferotemporal activation reflect emotional intensity and fear relevance. NeuroImage, 24, 1265-1270.

Schutter, D. J. L. G., Hofman, D., \& Van Honk, J. (2008). Fearful faces selectively increase corticospinal motor tract excitability: A transcranial magnetic stimulation study. Psychophysiology, 45, 345-348

Seibt, B., Neumann, R., Nussinson, R., \& Strack, F. (2008). Movement direction or change in distance? Self- and object-related approach-avoidance motions. Journal of Experimental Social Psychology, 44, 713-720.
Small, D. M., Gregory, M. D., Mak, Y. E., Gitelman, D., Mesulam, M. M., \& PARRISH, T. (2003). Dissociation of neural representation of intensity and affective valuation in human gustation. Neuron, 39, 701-711.

Tormos, J. M., Cañete, C., Tarazona, F., Catalá, M. D., PascualLeone Pascual, A., \& Pascual-Leone, A. (1997). Lateralized effects of self-induced sadness and happiness on corticospinal excitability. Neurology, 49, 487-491.

VAn Der Lubbe, R. H., \& Verleger, R. (2002). Aging and the Simon task. Psychophysiology, 39, 100-110.

Wassermann, E. M., Greenberg, B. D., Nguyen, M. B., \& Murphy, D. L. (2001). Motor cortex excitability correlates with an anxietyrelated personality trait. Biological Psychiatry, 50, 377-382.

\section{NOTES}

1. Premotor RT represents the time interval between the onset of an initiating cue and the onset of electromyography (EMG) activity in the corresponding muscle(s) surpassing a predetermined amplitude above baseline. Premotor RT (PRT) is a reflection of central processing (Basmajian \& De Luca, 1985). The time point demarcating the end of PRT to the beginning of overt movement (i.e., force onset) is termed motor RT (MT) and reflects the elapsed time associated with impulse propagation along the motor pathways and motor unit recruitment necessary for execution of the response (Li, Stevens, Kamper, \& Rymer, 2005). RT (which was used in the present study) represents the delay between the onset of the initiating cue and the onset of force production (PRT + MT).

2. Pleasant: 4290, 4694, 5626, 4689, 4677, 4658, 4490, 8190, 8080, $8034,8180,8030,8300,4651,4220,8193,5470,4664,8499,1650$, 5621, 4676, 4687, 4670, 4608, 4607, 4800, 8501, 4311, 4690, 4611, 8170, 4656, 4660, 4659, 8186, 8400, 4680, 5629. Unpleasant: 3053, $9901,9570,9405,3063,3266,6540,3000,3500,6350,3180,9910$, $3071,3102,3150,3069,2352.2,9410,6021,3120,2811,3060,3530$, $3140,2703,3016,3064,9300,3130,3010,3168,6415,3010,6022$, 3225, 3062, 3030, 6313, 9635.1. Neutral: 7031, 7140, 7006, 7235, 7705, $7491,2850,2190,5520,7041,7179,2038,7035,7110,5500,7055,5510$, 2480, 7100, 7217, 5740, 7050, 7950, 7020, 7000, 2397, 7025, 5731, 7038, 7009, 2570, 7080, 2840, 2880, 2890, 7185, 7004, 7233, 7161 .

3. IAPS normative valence and arousal ratings (Lang et al., 2005) for images shown during TMS and no-TMS trials. TMS trials: valence, pleasant, 7.01 (1.90); unpleasant, 1.80 (1.29); neutral, 4.97 (1.19); arousal, pleasant, 6.48 (2.19); unpleasant, 6.49 (2.32); neutral, 2.72 (1.98). No-TMS trials: valence, pleasant, 7.06 (1.69); unpleasant, 1.87 (1.30); neutral, 5.00 (1.16); arousal, pleasant, 6.47 (2.13); unpleasant, 6.54 (2.24); neutral, 2.64 (1.87).

(Manuscript received March 11, 2009; revision accepted for publication July 9, 2009.) 